\title{
Production of quarkonium and heavy flavour in ATLAS
}

\author{
Maximiliano Sioli*† \\ Bologna University and INFN \\ E-mail: sioliabo.infn.it
}

ATLAS has a wide program to study the production properties of conventional and exotic quarkonium, beauty, and charm bound states. We present the latest results on $J / \psi$ prompt and nonprompt components at $\sqrt{s}=7,8$ and $13 \mathrm{TeV}$, open charm production at at $\sqrt{s}=7 \mathrm{TeV}$, the ratio $f_{s} / f_{d}$ of $b$-quark fragmentation fractions at $\sqrt{s}=7 \mathrm{TeV}, B$ meson production at $\sqrt{s}=13 \mathrm{TeV}$ and the observation of $\Lambda_{b}^{0} \rightarrow \psi(2 S) \Lambda^{0}$ at $\sqrt{s}=8 \mathrm{TeV}$.

XIII International Conference on Heavy Quarks and Leptons

22-27 May, 2016

Blacksburg, Virginia, USA

* Speaker.

${ }^{\dagger}$ on behalf of the ATLAS Collaboration. 


\section{Introduction}

Measurements of heavy-quark production at the Large Hadron Collider (LHC) provide a means to test quantum chromodynamics (QCD) at the highest available collision energies in a region close to the boundary between the perturbative and non-perturbative regimes. Moreover experimental constraints on heavy-quark production are important in searches for new physics phenomena, for which they often represent an important background.

The ATLAS experiment has an extensive heavy flavour physics program. In this report we provide a selection of recent experimental results on charmonium and open charm production, namely the measurement of the differential cross sections of $J / \psi$ and $\psi(2 \mathrm{~S})$ production at 7 and $8 \mathrm{TeV}$ [1], the determination of the non-prompt $J / \psi$ fraction at $\sqrt{s}=13 \mathrm{TeV}$ [2] and the measurement of the $D^{* \pm}, D^{ \pm}$and $D_{s}^{ \pm}$production cross section at $\sqrt{s}=7 \mathrm{TeV}$ [3]. We also present some selected results on the study of $b$-hadron production and properties, namely the measurement of the ratio of $b$ quark fragmentation fractions $f_{s} / f_{d}$ at $\sqrt{s}=7 \mathrm{TeV}$ [4], the $B^{ \pm}$reconstruction at $\sqrt{s}=13 \mathrm{TeV}$ [5] and the observation of $\Lambda_{b}^{0} \rightarrow \psi(2 S) \Lambda^{0}$ at $\sqrt{s}=8 \mathrm{TeV}$ [6]. B meson CP violation and rare decays analyses were presented separately at this conference $[7,8]$.

\section{The ATLAS detector}

A detailed description of the ATLAS detector can be found in [9]. Here we outline the main components relevant to the analyses presented below.

ATLAS is a general-purpose detector consisting of an inner tracker, a calorimeter and a muon spectrometer. The Inner Detector (ID) has full coverage in $\phi$, covers the pseudorapidity range $|\eta|<$ 2.5 and operates inside an axial magnetic field of $2 \mathrm{~T}$ of a superconducting solenoid. It consists of a silicon pixel detector, a silicon microstrip detector (semiconductor tracker, SCT) and a transition radiation tracker (TRT). The inner-detector barrel (end-cap) parts consist of $3(2 \times 3)$ pixel layers, 4 $(2 \times 9)$ double-layers of single-sided SCT strips and $73(2 \times 160)$ layers of TRT straws. During the shutdown between LHC Run 1 and Run 2, the ID was upgraded with the Insertable B-Layer (IBL), an additional inner-most pixel layer which allows to enhance the tracking performances.

The ID is enclosed by a calorimeter system containing electromagnetic and hadronic sections. The calorimeter is surrounded by a large muon spectrometer (MS) in a toroidal magnet system. The MS consists of monitored drift tubes and cathode strip chambers, designed to provide precise position measurements in the bending plane in the range $|\eta|<2.7$. Momentum measurements in the muon spectrometer are based on track segments formed in at least two of the three precision chamber planes. Resistive Plate Chambers and Thin Gas Chambers are exploited for triggering events up to $\eta<2.4$. The combined muon performances of the ATLAS detector allow to measure momenta with a precision better than $10 \%$ up to $1 \mathrm{TeV}$. The resolution in the dimuon mass system is $\sim 50 \mathrm{MeV}(\sim 150 \mathrm{MeV})$ at the $J / \psi(\Upsilon(n S))$ resonance and the resolution in the $b$-hadron proper decay time is better than $100 \mathrm{fs}$.

The ATLAS detector has a multi-level trigger system. The hardware-based Level-1 trigger followed by the software-based High-Level Trigger stage (HLT), which runs reconstruction and calibration software, reducing the event rate to about $1 \mathrm{kHz}$ for data recording to mass storage. At Level-1, the muon triggers search for patterns of MS hits corresponding to dimuons passing various 
$p_{T}$ thresholds. Around these Level-1 hit patterns "Regions-of-Interest" (RoI) are defined that serve as seeds for the HLT muon reconstruction. The HLT uses dedicated algorithms to incorporate information from both the MS and the ID, achieving position and momentum resolution close to that provided by the offline muon reconstruction. For most of the analyses here presented dimuon triggers were used.

\section{3. $J / \psi$ and $\psi(2 S)$ production at 7 and $8 \mathrm{TeV}$}

At the LHC charmonium states can be produced either directly by short-lived QCD sources (prompt production), or by long-lived sources in the decay chains of beauty hadrons (non-prompt production). These can be separated experimentally using the distance between the proton-proton primary interaction and the decay vertex of the quarkonium state. The mechanism underlying the production of the prompt component is still a challenge for both theory and experiments while the non-prompt production is well understood within the perturbative QCD framework. Only the $\psi(2 S)$ vector meson is produced almost entirely in a prompt way and then it constitutes a valuable tool to test the production mechanisms of $J^{P C}=1^{--}$states.

The analysis here presented is based on $p p$ collisions taken at $\sqrt{s}=7 \mathrm{TeV}$ and $8 \mathrm{TeV}$ for a total integrated luminosities of $2.1 \mathrm{fb}^{-1}$ and $11.4 \mathrm{fb}^{-1}$ respectively. Events were selected using a trigger requiring two oppositely charged muon candidates, each passing the requirement $p_{T}>4 \mathrm{GeV}$. The offline analysis requires events with at least two muons identified by the muon spectrometer and with matching tracks reconstructed in the ID. Reconstructed candidates are obtained requiring that dimuon pairs have an invariant mass within the $2.6<m(\mu \mu)<4.0 \mathrm{GeV}$ window and in the kinematic range $p_{T}(\mu \mu)>8 \mathrm{GeV}$ and $|y(\mu \mu)|<2$, where $y(\mu \mu)$ is the rapidity of the dimuon system. Each dimuon candidate is weighted to correct for muon identification, trigger and reconstruction efficiencies and geometrical acceptance.

The prompt and non-prompt signal and background contributions were extracted from the data using a simultaneous two dimensional fit to the invariant mass of the dimuon and its "pseudo-proper decay time". The latter is defined as $\tau=L_{x y} m(\mu \mu) / p_{T}(\mu \mu)$ where $L_{x y}$ is the projection of the distance of the dimuon decay vertex from the primary vertex onto its transverse momentum. This
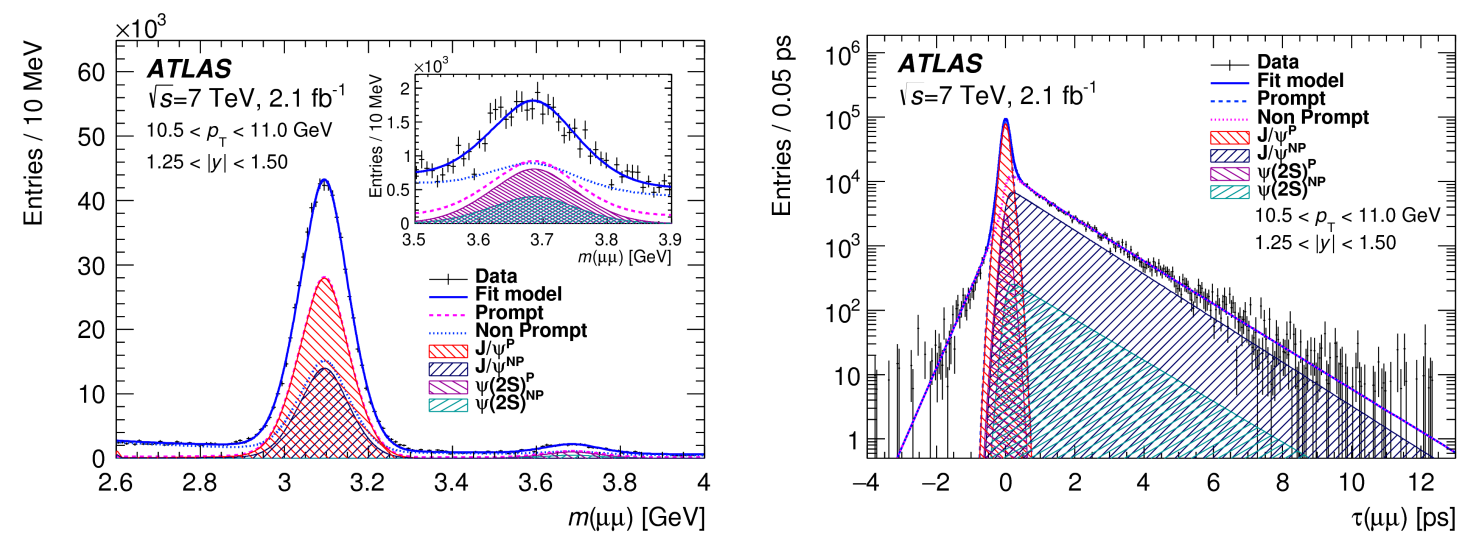

Figure 1: Projections of the fit result over the mass (left panel) and pseudo-proper decay time (right panel) distributions for data collected at $7 \mathrm{TeV}$ for one typical interval [1]. 

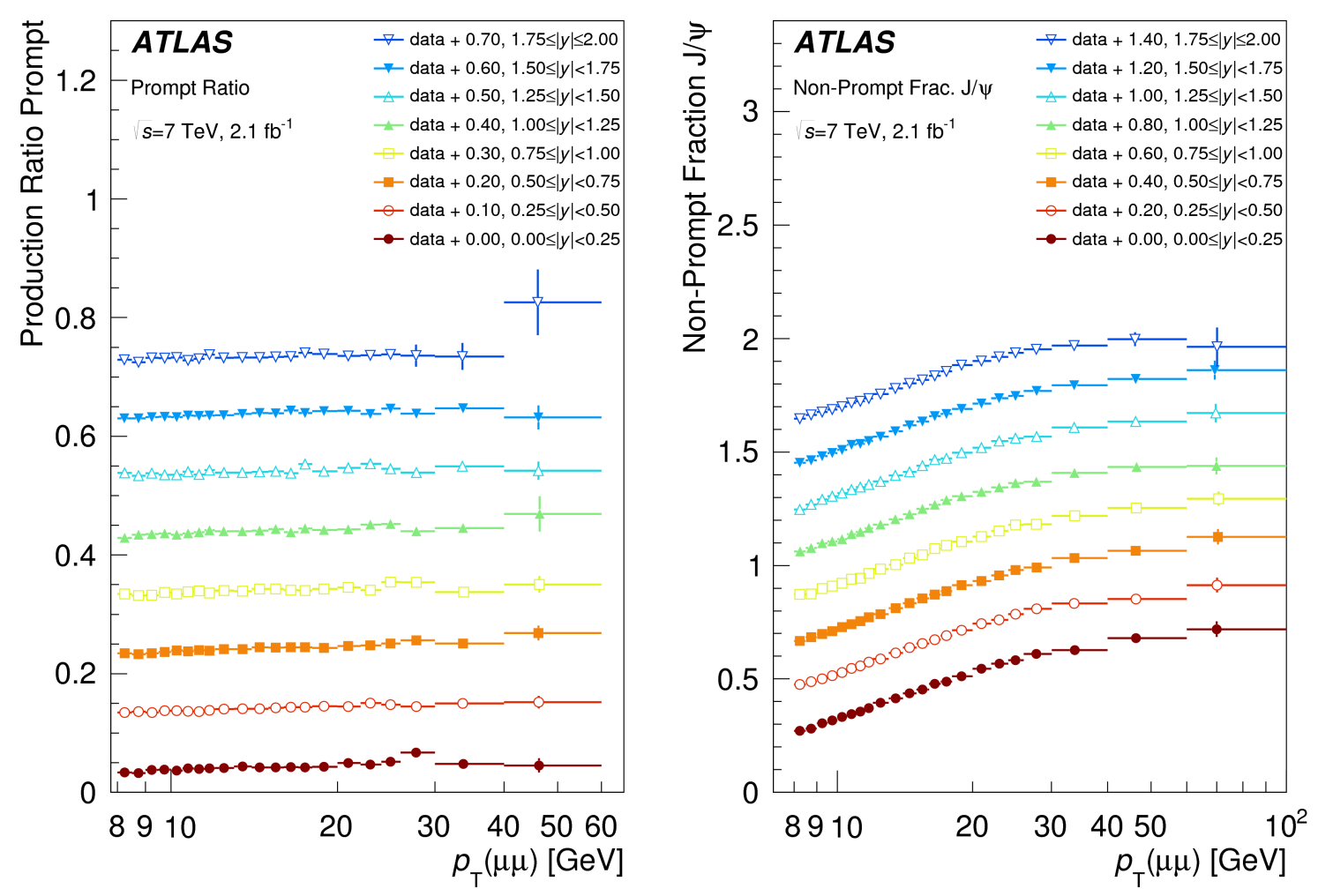

Figure 2: The ratio of $\psi(2 S)$ to $J / \psi$ production times dimuon branching fraction for prompt (left) and non-prompt fraction for $J / \psi$ (right) processes as a function of $p_{T}(\mu \mu)$ for each of the slices of rapidity. For each increasing rapidity slice, an additional factor of 0.1 is applied to the plotted points for visual clarity [1].

variable can be used to distinguish statistically between the non-prompt and prompt components. Signal mass models are described by the sum of a Crystal Ball shape and a Gaussian shape. The fit was performed separately in $22 \times 8$ in $\left(p_{T}(\mu \mu),|y(\mu \mu)|\right)$ intervals to take into account the resolution dependence on the kinematic region. In Fig. 1 we show the results of the fit projections on the invariant mass variable (left panel) and on the $\tau$ variable (right panel) for data taken at $\sqrt{s}=7 \mathrm{TeV}$.

ATLAS also provided differential prompt and non-prompt $J / \psi$ and $\psi(2 S)$ cross sections across a wide $p_{T}$ range as well as ratios of prompt $\psi(2 S) /$ prompt $J / \psi$ (Fig. 2, left panel) and prompt $J / \psi /$ non-prompt $J / \psi$. (Fig. 2, right panel). For the ratio measurements, similarly to the non-prompt fraction, the acceptance and efficiency corrections largely cancel, thus allowing a more precise measurement.

\section{Non-prompt $J / \psi$ fraction at $\sqrt{s}=13 \mathrm{TeV}$}

The non-prompt $J / \psi$ production fraction was also measured using the early $p p$ collision at $13 \mathrm{TeV}$, for a total integrated luminosity of approximately $6.4 \mathrm{pb}^{-1}$. Data were analysed using the same method of the previous analysis, selecting dimuon pairs in the region $p_{T}>8 \mathrm{GeV}$ and $|y|<$ 2.0. Simultaneous two-dimensional fits were performed in $11 \times 3\left(p_{T}(\mu \mu),|y(\mu \mu)|\right)$ bins using functional forms for signal and background similar to those used in the previous measurements. 
The dependence on the fit model represents the main contribution to the systematic uncertainty, followed by the uncertainty due to the trigger reconstruction efficiency.

The left panel of Fig. 3 shows the dependence of the non-prompt $J / \psi$ production fraction on the $p_{T}(\mu \mu)$ in the three rapidity bins considered in the analysis. In the right panel of Fig. 3 the non-prompt fraction is reported for different collision energies and in comparable rapidity ranges. A significant increase of the ratio is visible from $\sqrt{s}=2.76 \mathrm{TeV}$ to $\sqrt{s}=7 \mathrm{TeV}$ which could be ascribed to the different growth rate of $b$-hadrons and prompt $J / \psi$ cross sections with $\sqrt{s}$.
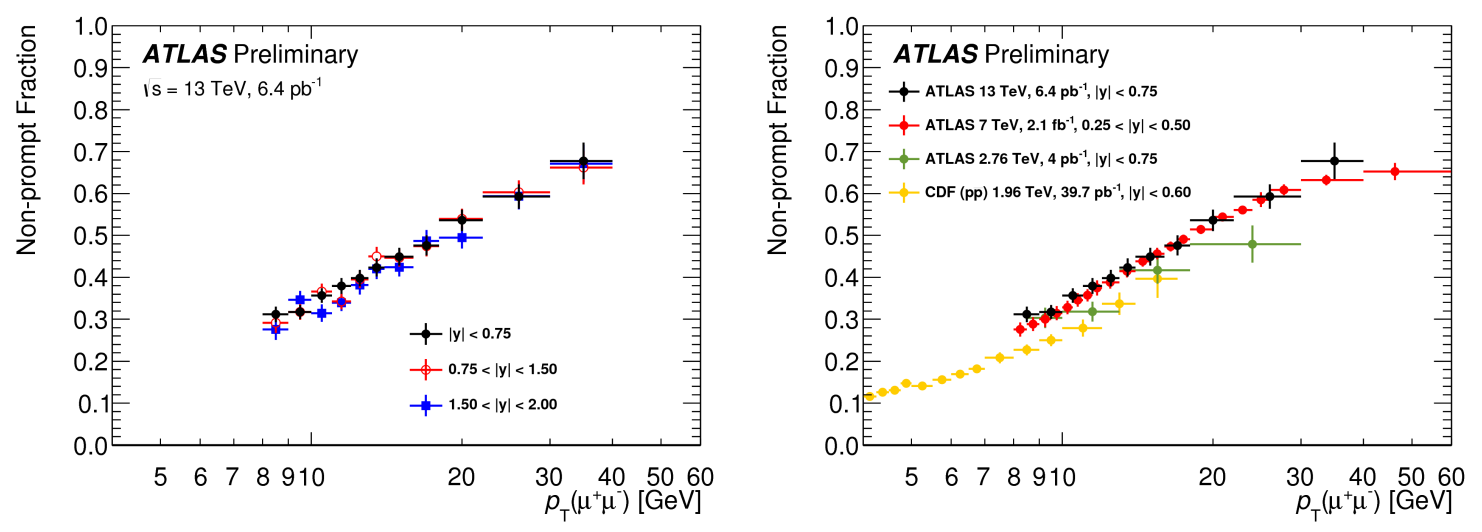

Figure 3: Left panel: measured non-prompt $J / \psi$ production fraction as a function of $p_{T}(\mu \mu)$ and in three intervals of $J / \psi$ rapidity. Right panel: measured non-prompt $J / \psi$ production fraction as a function of $p_{T}(\mu \mu)$ at different collision energies and in comparable rapidity intervals [2].

\section{5. $D^{* \pm}, D^{ \pm}$and $D_{s}^{ \pm}$production at $\sqrt{s}=7 \mathrm{TeV}$}

ATLAS measured the production of $D^{* \pm}, D^{ \pm}$and $D_{s}^{ \pm}$mesons in $p p$ collisions at $\sqrt{s}=7 \mathrm{TeV}$, using an integrated luminosity of $280 \mathrm{nb}^{-1}$. Charmed mesons were reconstructed in the range of transverse momentum $3.5<p_{T}(D)<100 \mathrm{GeV}$ and pseudorapidity $|\eta(D)|<2.1$ using tracks measured in the ID. As an example Fig. 4 (left panel) shows the case of the $D^{ \pm}$mesons reconstructed via the $D^{+} \rightarrow K^{-} \pi^{+} \pi^{+}$decay (and similarly for $D^{-}$). Besides the integrated cross section in the visible kinematic range, differential cross section $d \sigma / d p_{T}$ and $d \sigma / d|\eta|$ were obtained also for $D^{ \pm}$ (Fig. 4, right panel) and $D^{* \pm}$, which were compared to NLO QCD predictions.

Using the visible $D$ cross sections and an extrapolation to the full kinematic phase space, the total cross section of charm production in $p p$ collisions at $\sqrt{s}=7 \mathrm{TeV}$ was calculated. The resulting value [3]

$$
\sigma_{c \bar{c}}^{t o t}=\left[8.6 \pm 0.3(\text { stat }) \pm 0.7(\text { syst }) \pm 0.3(\text { lum }) \pm 0.2(\text { ff })_{-3.4}^{+3.8}(\text { extr })\right] \mathrm{mb}
$$

where the fourth uncertainty is due to the uncertainty of the fragmentation fractions and the last uncertainty is due to the extrapolation procedure, is in good agreement with a similar measurement performed by the ALICE experiment [10]. 

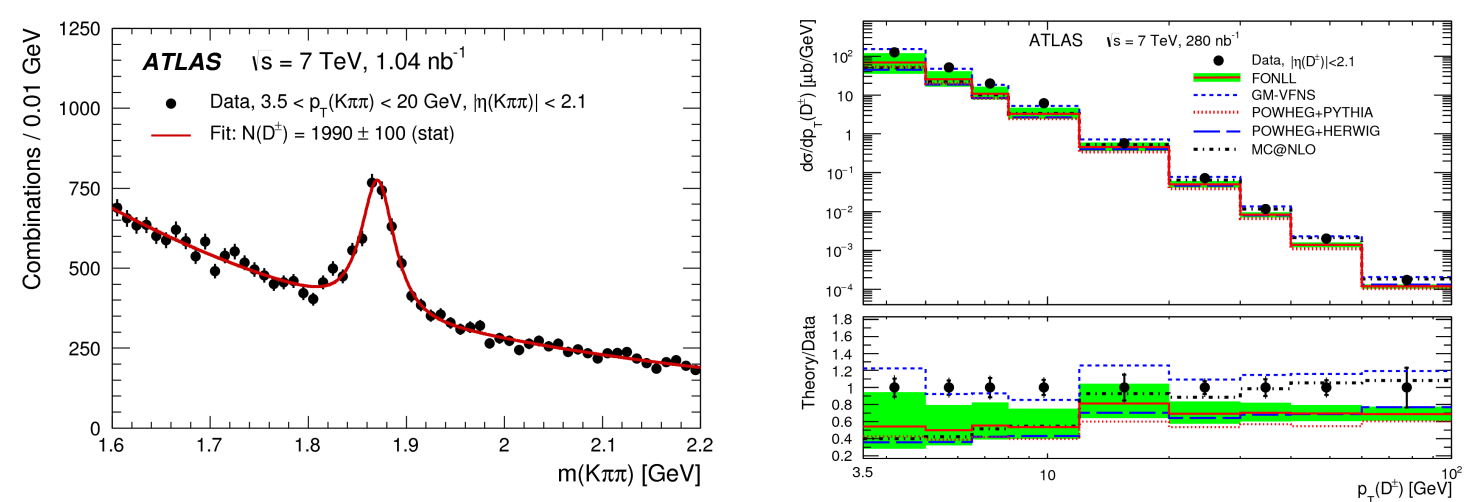

Figure 4: Left panel: distribution of the invariant mass $m(K \pi \pi)$ for $D^{ \pm}$meson candidates in a restricted kinematic region. The fit result is superimposed to data points. Right panel: corresponding differential cross sections of reconstructed $D^{ \pm}$meson as a function of $p_{T}$ compared to NLO QCD calculations and ratio of expectations to data (lower inset) [3].

\section{Ratio of $b$ quark fragmentation fractions $f_{s} / f_{d}$ at $\sqrt{s}=7 \mathrm{TeV}$}

The determination of $b$ quark fragmentation fractions $f_{s}\left(f_{d}\right)$, defined as the probability of an $\bar{b}$ quark to bind to an $s(d)$ quark, is important for measuring $b$-hadron cross sections and branching fractions, in particular for rare decays such as $B_{s}^{0} \rightarrow \mu^{+} \mu^{-}$.

The ratio $f_{s} / f_{d}$ is extracted from $B_{s}^{0}$ and $B_{d}^{0}$ meson yields. These are obtained from the measured $B_{s}^{0} \rightarrow J / \psi \phi$ and $B_{d}^{0} \rightarrow J / \psi K^{* 0}$ signal yields, $N_{B_{s}^{0}}$ and $N_{B_{d}^{0}}$, corrected for the branching fractions of the corresponding decays (obtained by PQCD predictions [11]) and for the relative acceptance and selection efficiency $\mathfrak{R}_{\text {eff }}$ obtained by Monte Carlo simulations. The $J / \psi, \phi$ and $K^{* 0}$ are reconstructed in their $J / \psi \rightarrow \mu^{+} \mu^{-}, \phi \rightarrow K^{+} K^{-}$and $K^{* 0} \rightarrow K^{+} \pi^{-}$final states, respectively. At
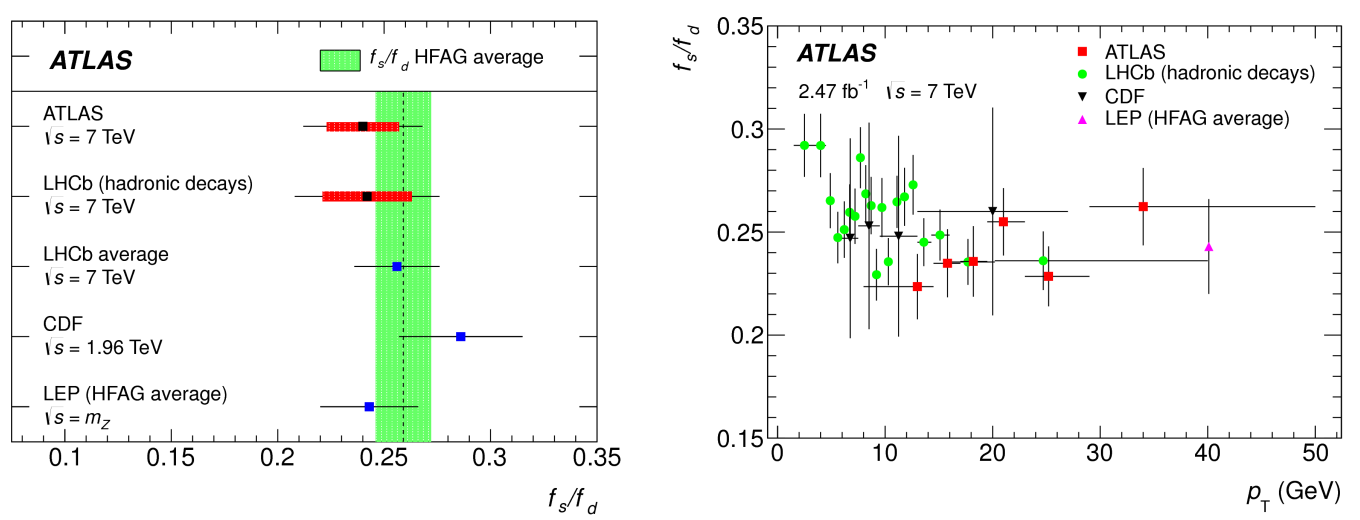

Figure 5: Integrated (left panel) and differential in $p_{T}$ (right panel) measurements of $f_{s} / f_{d}$ for CDF, LHCb and ATLAS (see [4] and references therein). The error bars show statistical and systematic errors added in quadrature. 
the end the extracted ratio can be expressed as:

$$
\frac{f_{s}}{f_{d}}=\frac{N_{B_{s}^{0}}}{N_{B_{d}^{0}}} \frac{\mathfrak{B}\left(B_{d}^{0} \rightarrow J / \psi K^{* 0}\right)}{\mathfrak{B}\left(B_{s}^{0} \rightarrow J / \psi \phi\right)} \frac{\mathfrak{B}\left(K^{* 0} \rightarrow K^{+} \pi^{-}\right)}{\mathfrak{B}\left(\phi \rightarrow K^{+} K^{-}\right)} \mathfrak{R}_{e f f} .
$$

The analysis of $2.47 \mathrm{fb}^{-1}$ of $p p$ collisions at $\sqrt{s}=7 \mathrm{TeV}$ provided a value $f_{s} / f_{d}=0.240 \pm$ 0.004 (stat) \pm 0.010 (syst) \pm 0.017 (theory) [4], where the main systematic uncertainty comes from the modeling of the signal and background parametrization for the $B$ meson decays. The measured value is in agreement with results from LEP, CDF and LHCb (Fig. 5, left panel). No particular $\eta$ or $p_{T}$ dependence was observed in the kinematic region of interest (Fig. 5, right panel).

\section{7. $B^{ \pm}$reconstruction at $\sqrt{s}=13 \mathrm{TeV}$}

The reconstruction of the $B^{ \pm}$mass at $13 \mathrm{TeV}$ is a good probe to assess the ID performance at the new energy conditions with increased pile-up and with the new IBL detector installed. The $B^{ \pm}$mass was determined in the $B^{ \pm} \rightarrow J / \psi\left(\mu^{+} \mu^{-}\right) K^{ \pm}$using the $3.2 \mathrm{fb}^{-1}$ of integrated luminosity collected in 2015. This analysis is also ancillary to future measurements of the $B$ meson decays when larger statistics will be accumulated.

$J / \psi$ candidates are reconstructed requiring two oppositely charged muons with $p_{T}>4 \mathrm{GeV}$ pointing to a common vertex, while kaon candidates are searched for with $p_{T}>3 \mathrm{GeV}$. An unbinned maximum-likelihood fit is then performed on the selected events to extract the $B^{ \pm}$mass. Since the mass resolution depends significantly on the rapidity region, the mass fit was independently performed in 16 rapidity $B^{ \pm}$intervals. The results show a good stability of the mass reconstruction over the full rapidity range of the ID. Overall this result shows a control of systematic uncertainty on the $p_{T}$ scale at per-mill level. Results are summarized in Table 1.

Table 1: Fitted $B^{ \pm}$mass values extracted from a weighted average of the fits to $B^{ \pm} \rightarrow J / \psi\left(\mu^{+} \mu^{-}\right) K^{ \pm}$ candidates in $y$ bins without and with $L_{x y}$ cut [5]. Also shown are the world average and LHCb values [12].

\begin{tabular}{l|c|c}
\hline \hline Fit & $B^{ \pm}$mass $[\mathrm{MeV}]$ & Fit error [MeV] \\
\hline Default Fit & 5279.31 & 0.11 (stat) \\
$L_{x y}>0.2 \mathrm{~mm}$ & 5279.34 & 0.09 (stat) \\
\hline World Average Fit & 5279.29 & 0.15 \\
LHCb & 5279.38 & 0.11 (stat) \pm 0.33 (syst) \\
\hline \hline
\end{tabular}

\section{Observation of $\Lambda_{b}^{0} \rightarrow \psi(2 S) \Lambda^{0}$ at $\sqrt{s}=\mathbf{8} \mathrm{TeV}$}

ATLAS made the first observation of the $\Lambda_{b}^{0} \rightarrow \psi(2 S) \Lambda^{0}$ decay and measured the branching ratio of the $\Lambda_{b}^{0} \rightarrow \psi(2 S) \Lambda^{0}$ and $\Lambda_{b}^{0} \rightarrow J / \psi \Lambda^{0}$ decays. The $J / \psi$ and $\psi(2 S)$ were reconstructed in the dimuon decay mode while the $\Lambda^{0} \rightarrow p \pi^{-}$decay was exploited for the $\Lambda^{0}$ baryon reconstruction. While the ratio of branching ratios of $B$ mesons decaying to $\psi(2 S) X$ and $J / \psi X$ are in the 0.5-0.8 range the only prediction available for $\Lambda_{b}^{0}$ gives a value of approximately 0.8 with an uncertainty of about $0.1[13]$. 
The invariant mass distributions for the combined sample of the selected $\Lambda_{b}^{0}$ and $\bar{\Lambda}_{b}^{0}$ is shown, in Fig. 6 for $\Lambda_{b}^{0} \rightarrow J / \psi \Lambda^{0}$ (left plot) and $\Lambda_{b}^{0} \rightarrow \psi(2 S) \Lambda^{0}$ (right plot). In the signal reconstruction, reflection from $B^{0}$ decays to the $\Lambda_{b}^{0}$ sample was taken into account by simultaneously fitting the $m_{J / \psi \Lambda^{0}}$ and $m_{J / \psi K_{S}^{0}}$ distributions (and similarly for $\psi(2 S)$ ). The $\Lambda_{b}^{0}$ baryons are reconstructed with transverse momentum $p_{T}>10 \mathrm{GeV}$ and pseudorapidity $|\eta|<2.1$.

The ratio of the two $\Lambda_{b}^{0}$ decay modes are calculated as

$$
\frac{\Gamma\left(\Lambda_{b}^{0} \rightarrow \psi(2 S) \Lambda^{0}\right)}{\Gamma\left(\Lambda_{b}^{0} \rightarrow J / \psi \Lambda^{0}\right)}=\frac{N_{c o r}\left(\Lambda_{b}^{0} \rightarrow \psi\left(\mu^{+} \mu^{-}\right) \Lambda^{0}\right)}{N_{c o r}\left(\Lambda_{b}^{0} \rightarrow J / \psi\left(\mu^{+} \mu^{-}\right) \Lambda^{0}\right)} \cdot \frac{\mathfrak{B}\left(J / \psi \rightarrow l^{+} l^{-}\right)}{\mathfrak{B}\left(\psi(2 S) \rightarrow l^{+} l^{-}\right)}
$$

where $\mathfrak{B}$ is the branching fraction of the corresponding charmonium decay to a lepton pair and $N_{c o r}$ is the number of $\Lambda_{b}^{0}$ signal events in the two decay modes, after correcting for detector effects and selection efficiencies. The measured branching ratio with $20.6 \mathrm{fb}^{-1}$ of collision data is $0.501 \pm 0.033$ (stat) \pm 0.016 (syst) $\pm 0.011(\mathfrak{B}$ ), where the main source of systematic uncertainty is due to the signal extraction procedure and the uncertainties related to the charmonium branching fractions $(\mathfrak{B})$ is quoted separately.
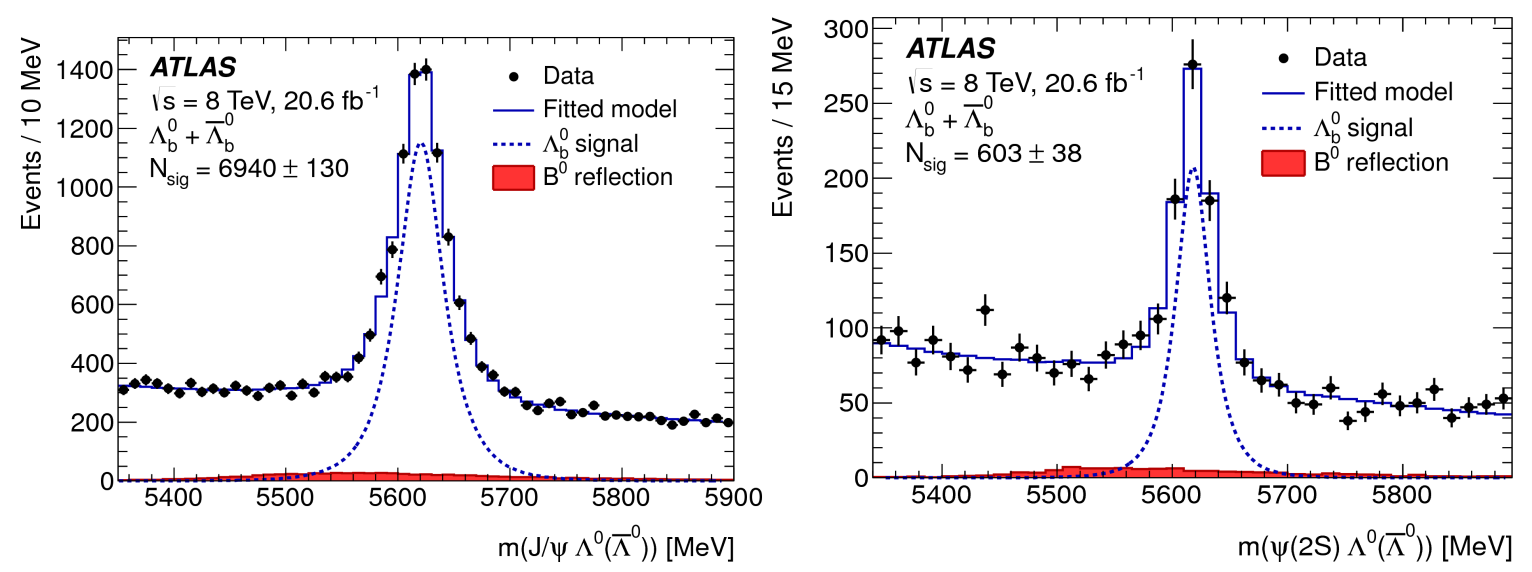

Figure 6: The invariant mass distributions for the combined sample of the selected $\Lambda_{b}^{0}$ and $\bar{\Lambda}_{b}^{0}$ for $\Lambda_{b}^{0} \rightarrow$ $J / \psi \Lambda^{0}$ (left plot) and $\Lambda_{b}^{0} \rightarrow \psi(2 S) \Lambda^{0}$ (right plot) [6].

\section{Conclusions}

We presented some selected results from Run 1 at LHC performed by the ATLAS experiment on quarkonium and $b$-hadron production and decays: the measurement of the differential cross sections of $J / \psi$ and $\psi(2 \mathrm{~S})$, the measurement of the $D^{* \pm}, D^{ \pm}$and $D_{s}^{ \pm}$production cross section, the measurement of the ratio of $b$ quark fragmentation fractions $f_{s} / f_{d}$ and the observation of $\Lambda_{b}^{0} \rightarrow$ $\psi(2 S) \Lambda^{0}$ decay. From early Run 2 data we reported the determination of the non-prompt $J / \psi$ fraction and the $B^{ \pm}$reconstruction. These results offer the possibility to test and tune the Monte Carlo generators and help to shed new light on the production of QCD states at the boundary of the perturbative regime in unexplored energy regions. The ATLAS heavy physics program operates in synergy with other LHC experiments and will profit of the large statistics which is being collected in Run 2 to improve previous measurements and provide new results in the forthcoming future. 


\section{References}

[1] ATLAS Collaboration, Measurement of the differential cross-sections of prompt and non-prompt production of $\mathrm{J} / \psi$ and $\psi(2 \mathrm{~S})$ in pp collisions at $\sqrt{s}=7$ and $8 \mathrm{TeV}$ with the ATLAS detector, Eur. Phys. J. C 76 (2016) no. 5, 283, arXiv:1512.03657 [hep-ex].

[2] ATLAS Collaboration, Measurement of the differential non-prompt $J / \psi$ production fraction in $\sqrt{s}=13 \mathrm{TeV} p$ p collisions at the ATLAS experiment, ATLAS-CONF-2015-030, http://cds.cern. ch/record/2037967.

[3] ATLAS Collaboration, Measurement of $D^{* \pm}, D^{ \pm}$and $D_{s}^{ \pm}$meson production cross sections in pp collisions at $\sqrt{s}=7$ TeV with the ATLAS detector, Nucl. Phys. B 907 (2016) 717, arXiv:1512.02913 [hep-ex].

[4] ATLAS Collaboration, Determination of the ratio of b-quark fragmentation fractions $f_{s} / f_{d}$ in $p p$ collisions at $\sqrt{s}=7 \mathrm{TeV}$ with the ATLAS detector, Phys. Rev. Lett. 115 (2015) no. 26, 262001, arXiv:1507.08925 [hep-exp].

[5] ATLAS Collaboration, $B^{ \pm}$mass reconstruction in $B^{ \pm} \rightarrow J / \psi K^{ \pm}$decay at ATLAS at 13 TeV pp collisions at the LHC, ATLAS-CONF-2015-064, http: / / cds . cern. ch/record/2114830.

[6] ATLAS Collaboration, Measurement of the branching ratio $\Gamma\left(\Lambda_{b}^{0} \rightarrow \psi(2 S) \Lambda^{0}\right) / \Gamma\left(\Lambda_{b}^{0} \rightarrow J / \psi \Lambda^{0}\right)$ with the ATLAS detector, Phys. Lett. B 751 (2015) 63, arXiv:1507.08202 [hep-ex].

[7] E. Bouhova-Thacker, Mixing and CP violation in the $B_{d}$ and $B_{s}$ systems at ATLAS, to appear in these proceedings.

[8] J. Günther, $B_{d / s} \rightarrow \mu^{+} \mu^{-}$in ATLAS, to appear in these proceedings.

[9] ATLAS Collaboration, The ATLAS Experiment at the CERN Large Hadron Collider, JINST 3 (2008) S08003.

[10] B. Abelev et al. [ALICE Collaboration], Measurement of charm production at central rapidity in proton-proton collisions at $\sqrt{s}=2.76 \mathrm{TeV}$, JHEP 1207 (2012) 191.

[11] X. Liu, W. Wang and Y. Xie, Penguin pollution in $B \rightarrow J / \psi V$ decays and impact on the extraction of the $B_{s}-\bar{B}_{s}$ mixing phase, Phys. Rev. D 89 (2014) no.9, 094010.

[12] R. Aaij et al. [LHCb Collaboration], Measurement of b-hadron masses, Phys. Lett. B 708 (2012) 241.

[13] T. Gutsche et al., Polarization effects in the cascade decay $\Lambda_{b} \rightarrow \Lambda\left(\rightarrow p \pi^{-}\right)+J / \psi\left(\rightarrow l^{+} l^{-}\right)$in the covariant confined quark model, Phys. Rev. D 88 (2013) no. 11, 114018. 